\title{
Case - Bilateral and recurrent pediatric cystic nephroma associated with DICER1 mutation
}

Cindy Pelletier Caron ${ }^{1}$; Bruno Turcotte ${ }^{1}$; Marie-Claude Pelland-Marcotte ${ }^{2}$; Kris Ann P. Schultz ${ }^{3}$; Isabelle Harvey ${ }^{4}$; Stéphane Bolduc ${ }^{1,5}$

${ }^{1}$ Division of Urology, Department of Surgery, CHU de Québec-Université Laval, Quebec City, QC, Canada; ${ }^{2}$ Division of Hemato-oncology, Department of Pediatrics, CHU de Québec-Université Laval, Quebec City, QC, Canada; ${ }^{3}$ International PPB/DICER1 Registry, Cancer and Blood Disorders, Children's Minnesota, Minneapolis, MN, United States; ${ }^{4}$ Division of Pathology, Department of Laboratory Medicine, CHU de Québec-Université Laval, Quebec City, QC, Canada; ${ }^{5}$ Division of Regenerative Medicine, CHU de Québec-Université Laval Research Center, Quebec City, QC, Canada

Cite as: Caron CP, Turcotte B, Pelland-Marcotte M-C, et al. Case - Bilateral and recurrent pediatric cystic nephroma associated with DICER1 mutation. Can Urol Assoc J 2020 October 27; Epub ahead of print. http://dx.doi.org/10.5489/cuaj.6836

Published online October 27, 2020

$* * *$

\section{Introduction}

Pathogenic variation in DICER1 predisposes to a variety of benign and malignant neoplasms, including pleuropulmonary blastoma (PPB) and cystic nephroma (CN). Other rare tumors that can be seen include Sertoli-Leydig cell, Wilms, thyroid cancer, and certain childhood brain tumors. More recently, anaplastic sarcoma of the kidney (ASK) has also been described. ${ }^{1,2}$ DICER1 pathogenic variation is inherited in an autosomaldominant fashion with low to reduced penetrance (15\%). With this case report, we review the current literature on this rare syndrome, with a focus on urological concerns.

\section{Case presentation}

The patient is a nine years old boy who has been followed since birth as a DICER1 mutation carrier. His family history revealed a maternal cousin with a pituitary blastoma

at eight months of age. That cousin, his mother, our patient and his mother, were found to be all carriers of the DICER1 mutation c.2379T $>\mathrm{G}$ [p.Y793X]. During his recommended follow-up, two lesions were discovered at 13 months old: one $38 \times 28 \times 34 \mathrm{~mm}$ in the right upper pulmonary lobe and a 41x34x36 mm lesion between the middle and upper pole of the left kidney. First, a right lobectomy was done, and the pathology revealed a Type I PPB. One month later, a left partial nephrectomy was performed. The pathologist confirmed a $\mathrm{CN}$ with negative margins. The patient received a 40 -week protocol of 
adjuvant chemotherapy for his Type I PPB (Vincristine, Dactinomycin and Cyclophosphamide). After seven years of follow-up, the patient developed a new $27 \times 30 \times 32 \mathrm{~mm}$ multicystic lesion on the inferior pole of the contralateral right kidney. A second partial nephrectomy was done in August 2019. The pathology again showed a CN with negative margins. At his first post-operative follow-up, two months later, a new third renal lesion was identified. A $12 \mathrm{~mm}$ simple cyst lesion on the superior pole of the right kidney was found on ultrasound (U/S). At the three-month follow-up, the cyst had progressed to $16 \mathrm{~mm}$ and had more complex features with small septations. Another U/S was obtained two months later and showed significant growth of the lesion, now measuring $24 \times 24 \times 27 \mathrm{~mm}$. After a discussion with the family and the multidisciplinary team, including members of the DICER1 International PPB/DICER1 Registry, the decision was made to resect the lesion. Despite the risk of fibrosis due to multiple interventions, we elected to perform a nephron-sparing surgery, with minimal morbidity, while avoiding malignant transformation. He underwent an uneventful right partial nephrectomy (enucleation type resection). He was discharged on post-op day two, and his creatinine remained normal to his baseline. The pathology review showed an unusual CN with negative margins. The cysts showed cuboidal lining epithelium with frequent hobnailing. Cystic septa are generally hypocellular and fibrous, but focally showed layer of immatures subepithelial cells, and made this pathology similar to type-1 PPB (figure 2)

The institutional review board approved the study with fully informed parental consent and minor assent for the use of tumour material for biological studies.

\section{Discussion}

Germline pathogenic DICER1 variation predisposes to a variety of cancers, including PPB, Sertoli-Leydig cell tumour, ASK and childhood brain tumours, as well as dysplasia and benign conditions such as thyroid nodules. Some benign conditions, such as cystic nephroma, have a risk of malignant transformation. [3, 4]

Mutations in the DICER1 gene are affecting RNA expression. [5] As postulated by the two-hit hypothesis, tumours appear because one copy of the DICER1 gene is affected by a predisposing germline mutation (usually inherited in an autosomal dominant fashion, although $10-20 \%$ of mutations are sporadic), and the other copy is affected by an acquired, somatic, tumour-specific mutation. [4] In United-States, approximately 1 in 10,600 people carries a pathogenic variant of the mutation, but it is estimated that only $20 \%$ of carriers will develop a neoplasm by 50 years of age. $[4,6,7]$ Approximately $70 \%$ of PPBs and pediatric CNs are attributable to pathogenic DICER 1 mutations. [8] Genetic counselling and consideration of testing are recommended for children and relatives of individuals with DICER1 pathogenic variation. Surveillance recommendations are available and include chest, thyroid and abdominal imaging, in 
addition to individual, family and provider education. $[9,10]$ The actual surveillance recommendations for kidney imaging of a patient with DICER1 mutation is an U/S every six months for the first eight years of life, and every year until 12 years old $[8,10,11]$ although the clinical utility and cost-effectiveness of this surveillance strategy has not been formally studied yet. To date, the literature is scarce concerning the follow-up recommendations after resection of $\mathrm{CN}$ or for stable $\mathrm{CN}$. However, at least an abdominal $\mathrm{U} / \mathrm{S}$ every six months should be obtained for the first few years after a $\mathrm{CN}$ diagnosis. [8, $10,11]$ Our practice has been to perform U/S every three months for children with a history of CN.

Individuals with pathogenic germline DICER1 variation or a history of DICER1related condition(s) including $\mathrm{CN}$, renal sarcoma, PPB or sex cord-stromal tumours, are eligible for the International PPB/DICER1 Registry. The International PPB/DICER1 Registry provides central pathology review and shares information with families and treating health care providers.

$\mathrm{CN}$ is a benign renal tumour affecting adults and children where it can be associated with DICER1 mutation. In childhood, males are more affected by $\mathrm{CN}$ while women are more affected in adulthood. [12] Diagnostic is usually made within the first four years of life but has been made in teenagers up to 14 years of age. Symptoms can be a sensation of an abdominal mass, abdominal pain, hematuria or urinary tract infection. The most common imaging modality used is abdominal $\mathrm{U} / \mathrm{S}$, which demonstrates an intrarenal complex mass, hypo or avascular, with a capsule and multilocular cysts separated by septae. At this point, it can be challenging to make the difference between a $\mathrm{CN}$ and a more differentiated cyst, based solely on imaging. The only way to confirm it is on the pathological specimen of resection. [8] Thus far, the risk of DICER1-associated $\mathrm{CN}$ for malignant transformation appears low; however, most cases of $\mathrm{CN}$ have been resected. In addition to the prevention of malignant transformation, early resection may also be nephron sparing. Unfortunately, however, children with DICER1-related CN have a risk for bilateral disease. To date, the actual literature suggests removing larger or symptomatic cysts with surgery. Surgery is the only management option for $\mathrm{CN}$, preoperative neoadjuvant chemotherapy is not suggested. [13] Smaller CN lesions could be followed using only U/S, but more complex features should raise suspicions and lead to anatomical imaging. [2] A recent case report and research by McGill University, addressed the risk of transformation of $\mathrm{CN}$ in ASK. It seems that ASK may arise from a pre-existing $\mathrm{CN}$, a continuum analogous to the type I PPB cysts becoming a type II or III PPB. $[1,2,14,15]$ With this new information in mind, the question of early diagnosis and removal of smaller cysts emerge. In this case, we highlight the challenge of 
managing $\mathrm{CN}$ in the setting of genetic predisposition, which in this case included bilateral and recurrent $\mathrm{CN}$.

\section{Conclusions}

This case highlights the dilemma between removal versus follow-up of small kidney cystic masses in patients with DICER1 pathogenic variation. With the present case, the option of surveillance was initially adopted, but resection became necessary as the $\mathrm{CN}$ rapidly increased in size. 
Case: Pediatric cystic nephroma associated with DICER1 mutation

\section{References}

1. Wu, M.K., et al., Tumor progression in DICER1-mutated cystic nephromawitnessing the genesis of anaplastic sarcoma of the kidney. Hum Pathol, 2016. 53: p. 114-20.

2. Wu, M.K., et al., Evolution of Renal Cysts to Anaplastic Sarcoma of Kidney in a Child With DICER1 Syndrome. Pediatr Blood Cancer, 2016. 63(7): p. 1272-5.

3. Abbo, O., et al., Wilms tumor, pleuropulmonary blastoma, and DICER1: case report and literature review. World J Surg Oncol, 2018. 16(1): p. 164.

4. Guillerman, R.P., W.D. Foulkes, and J.R. Priest, Imaging of DICER1 syndrome. Pediatr Radiol, 2019. 49(11): p. 1488-1505.

5. Dillman, J.R., et al., Hereditary Renal Cystic Disorders: Imaging of the Kidneys and Beyond. Radiographics, 2017. 37(3): p. 924-946.

6. Kim, J., et al., The prevalence of DICER1 pathogenic variation in population databases. Int J Cancer, 2017. 141(10): p. 2030-2036.

7. Stewart, D.R., et al., Neoplasm Risk Among Individuals With a Pathogenic Germline Variant in DICER1. J Clin Oncol, 2019. 37(8): p. 668-676.

8. Faure, A., et al., DICER1 pleuropulmonary blastoma familial tumour predisposition syndrome: What the paediatric urologist needs to know. J Pediatr Urol, 2016. 12(1): p. 5-10.

9. Schultz, K.A.P., et al., DICER1 and Associated Conditions: Identification of Atrisk Individuals and Recommended Surveillance Strategies. Clin Cancer Res, 2018. 24(10): p. 2251-2261.

10. Bauer, A.J., et al., DICER1 and Associated Conditions: Identification of At-risk Individuals and Recommended Surveillance Strategies-Response. Clin Cancer Res, 2019. 25(5): p. 1689-1690.

11. Srinivasan, A.S., et al., Imaging surveillance for children with predisposition to renal tumors. Pediatr Radiol, 2019. 49(11): p. 1453-1462.

12. Granja, M.F., et al., Multilocular Cystic Nephroma: A Systematic Literature Review of the Radiologic and Clinical Findings. AJR Am J Roentgenol, 2015. 205(6): p. 1188-93.

13. Kurian, J.J., S. Jehangir, and A. Korula, Multiloculated Cystic Renal Tumors of Childhood: Has the Final Word Been Spoken. J Indian Assoc Pediatr Surg, 2018. 23(1): p. 22-26.

14. Doros, L.A., et al., DICER1 mutations in childhood cystic nephroma and its relationship to DICER1-renal sarcoma. Mod Pathol, 2014. 27(9): p. 1267-80.

15. Wu, M.K., et al., Anaplastic sarcomas of the kidney are characterized by DICER1 mutations. Mod Pathol, 2018. 31(1): p. 169-178. 


\section{Figures and Tables}

Fig. 1. Cystic nephromas in DICER-1 patient. (A) A first lesion in the middle pole of the left kidney was resected in 2012. (B) A second cystic nephroma was resected in 2019 in the right kidney. (lower pole). (C) A third cystic mass resected in April 2020, which appeared to be an unusual cystic nephroma.

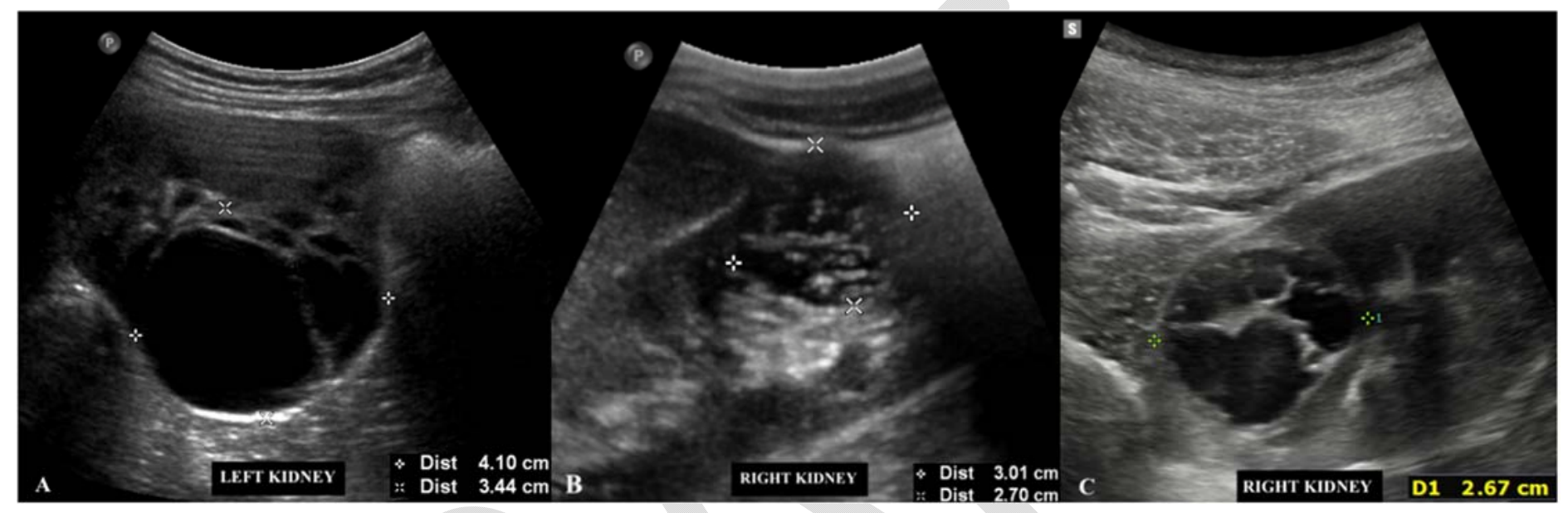

Fig. 2. Third cystic lesion resected: unusual cystic nephroma. (A) Macroscopic pathology (multicystic multilocular lesion with thin septa and translucent content). (B) Microscopic pathology (cuboidal lining epithelium with frequent hobnailing).

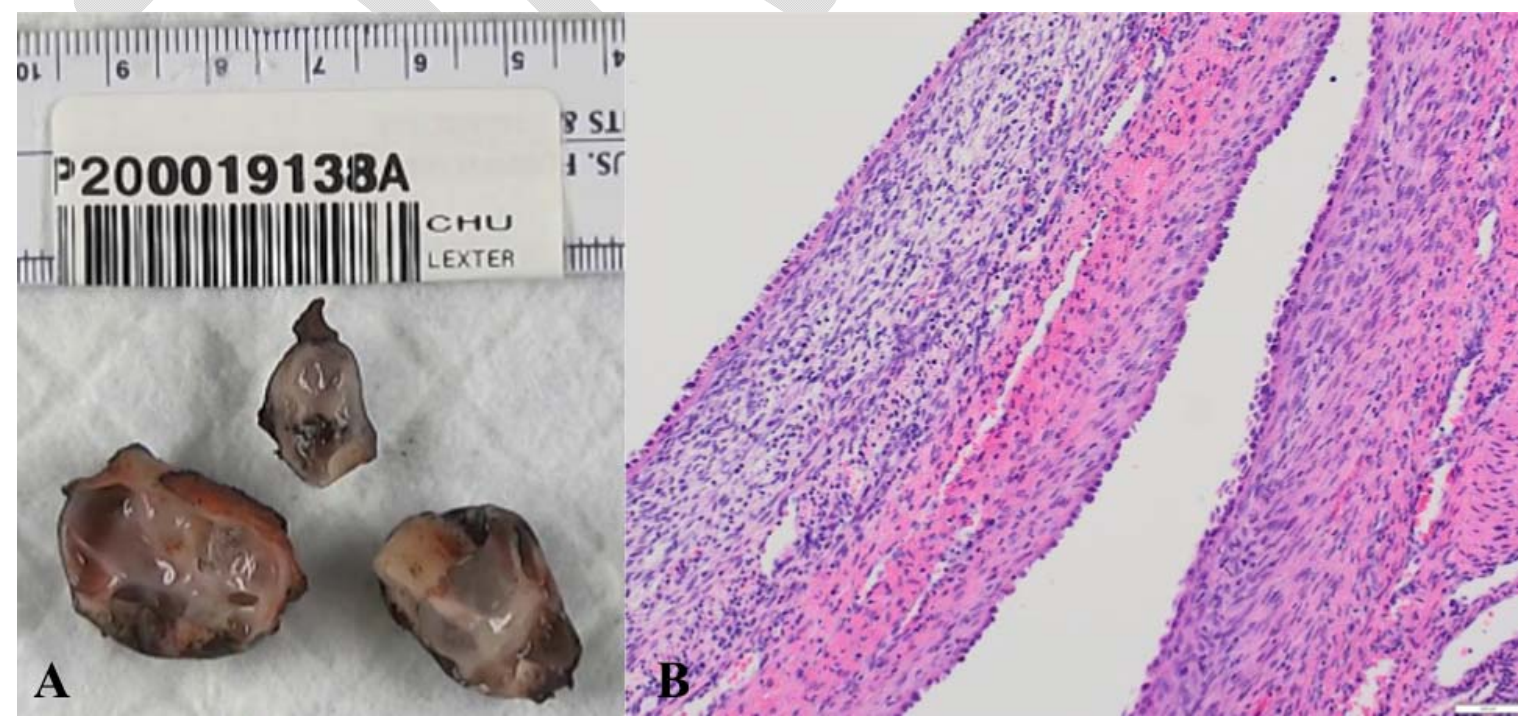

